\title{
Fault Diagnosis of Overflow Valve Based on Trispectrum
}

\author{
Wenbing Wu \\ Nanchang Normal University, Nanchang, China \\ Email: wwbysq@fjnu.edu.cn
}

How to cite this paper: Wu, W.B. (2020) Fault Diagnosis of Overflow Valve Based on Trispectrum. World Journal of Engineering and Technology, 8, 765-773. https://doi.org/10.4236/wjet.2020.84055

Received: October 5, 2020

Accepted: November 17, 2020

Published: November 20, 2020

Copyright ( 2020 by author(s) and Scientific Research Publishing Inc. This work is licensed under the Creative Commons Attribution International License (CC BY 4.0).

http://creativecommons.org/licenses/by/4.0/

\begin{abstract}
The high-order spectrum can effectively remove Gaussian noise. The three-spectrum and its slices represent random signals from a higher probability structure. It can not only qualitatively describe the linearity and nonlinearity of vibration signals closely related to mechanical failures, Gaussian and non-Gaussian Performance, and can greatly improve the accuracy of mechanical fault diagnosis. The two-dimensional slices of trispectrum in normal and fault states show different peak characteristics. 2-D wavelet multi-level decomposition can effectively compress 2 -D array information. Least squares support vector machine can obtain the global optimum under limited samples, thus avoiding the local optimum problem, and has the advantage of reducing computational complexity. In this paper, 2-D wavelet multi-level decomposition is used to extract features of trispectrum 2-D slices, and input LSSVM to diagnose the fault of the pressure reducing valve, which has achieved good results.
\end{abstract}

\section{Keywords}

Speed Control Valve, Trispectrum Two-Dimensional Slice, Two-Dimensional Wavelet, Least Square Support Vector Machine, Fault Diagnosis

\section{Introduction}

High-order spectra can effectively remove Gaussian noise. The trispectrum and its slices can not only qualitatively describe the linearity and nonlinearity of vibration signals closely related to mechanical faults, and the performance of Gaussian and non-Gaussian, but also greatly improve the diagnosis accuracy of mechanical faults [1]. In this paper, on the basis of obtaining the trispectrum 2-D slice spectrum of the vibration signal of the speed control valve, the 2-D wavelet multi-level decomposition is used to extract the features of the bi-coherent spec- 
trum, and the least square support vector machine is input to judge the faults.

\section{Data Acquisition and Dynamic Test Program}

The software virtual instrument software development tool LabVIEW [2] is used for the acquisition and processing of vibration signals. Using LabVIEW and PCI-6014 data acquisition card and an acceleration sensor, the vibration signals of the overflow valve (Figure 1) under normal and fault conditions are collected in sequence. In each measurement, the oil pressure is divided into 5 pressure levels from $1 \mathrm{MPa}$ to $5 \mathrm{MPa}$. The sampling frequency is $250 \mathrm{~Hz}$, the reading frequency is $125 \mathrm{~Hz}$, and the sampling process time is about $2 \mathrm{~min}$. In this experiment, 18 sets of data, a total of 36 sets were collected in the normal working state and fault state of the overflow valve. The number of data used in this experiment of every set is 1024. The experimental data acquisition program is shown in Figure 2 [2]. Due to the influence of various external and internal factors of the system during the test process, many unwanted components will inevitably be mixed in the output process, so it is necessary to preprocess the obtained data to eliminate the interference noise mixed in the signal. This paper uses the median method to preprocess the collected vibration signals.

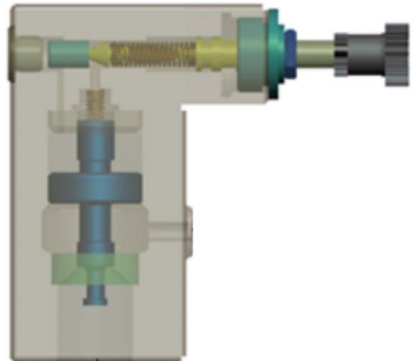

Figure 1. Overflow valve.

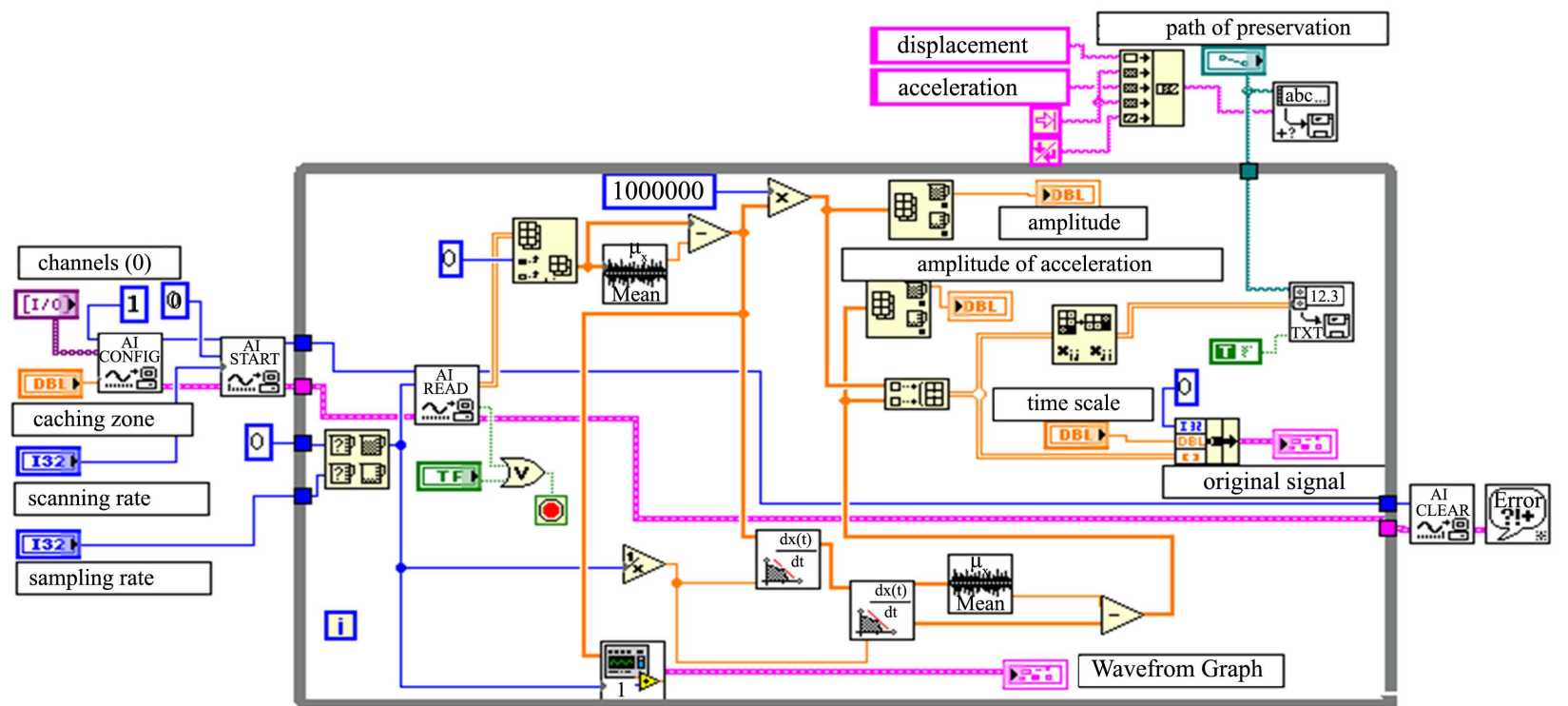

Figure 2. Data acquisition program diagram. 


\section{Two-Dimensional Slice of Trispectrum}

Let the high-order cumulant $c_{k x}\left(\tau_{1}, \tau_{2}, \cdots, \tau_{k-1}\right)$ be absolutely summable:

$$
\sum_{\tau_{1}=-\infty}^{\infty} \cdots \sum_{\tau_{k-1}=-\infty}^{\infty}\left|c_{k x}\left(\tau_{1}, \cdots, \tau_{k-1}\right)\right|<\infty
$$

Then the k-order cumulant spectrum is defined as the $k-1$ dimensional Fourier transform of the k-order cumulant, that is:

$$
S_{k x}\left(\omega_{1}, \cdots, \omega_{k-1}\right)=\sum_{\tau_{1}=-\infty}^{\infty} \cdots \sum_{\tau_{k-1}=-\infty}^{\infty} c_{k x}\left(\tau_{1}, \cdots, \tau_{k-1}\right) \exp \left[-j \sum_{i=1}^{k-1} \omega_{i} \tau_{i}\right]
$$

Then trispectrum is:

$$
T_{x}\left(\omega_{1}, \omega_{2}, \omega_{3}\right)=\sum_{\tau_{1}=-\infty}^{\infty} \sum_{\tau_{2}=-\infty}^{\infty} \sum_{\tau_{3}=-\infty}^{\infty} c_{4 x}\left(\tau_{1}, \tau_{2}, \tau_{3}\right) \mathrm{e}^{-j\left(\omega_{1} \tau_{1}+\omega_{2} \tau_{2}+\omega_{3} \tau_{3}\right)}
$$

After the AR model is established, the three spectrum can also be expressed in the form of frequency characteristic function:

$$
T\left(\omega_{1}, \omega_{2}, \omega_{3}\right)=\gamma_{4 a} H\left(\omega_{1}\right) H\left(\omega_{2}\right) H\left(\omega_{3}\right) H^{*}\left(\omega_{1}+\omega_{2}+\omega_{3}\right)
$$

where $H(\omega)=\frac{1}{1+\sum_{i=1}^{p} \phi_{i} \mathrm{e}^{-j i \omega}}$ is transfer Function,

$$
\gamma_{4 a}=E\left\{a^{4}(n)\right\}-3\left[E\left\{x^{2}(n)\right\}\right]^{2}
$$

is tosis, $a(n)$ is an independent and identically distributed non-Gaussian stochastic process.

The triple spectrum can reflect the steepness of the system's deviation from the Gaussian process, and can be used to detect and characterize the cubic phase coupling of the system. The mode of the trispectrum is a function of three frequency variables, which needs to be described in a four-dimensional space.

In order to obtain the trispectrum graph, a moving ball and movable surface can usually be used to display the amplitude of each point of the three-spectrum in the four-dimensional space, as shown in Figure 3 and Figure 4 [3] [4] [5] [6].

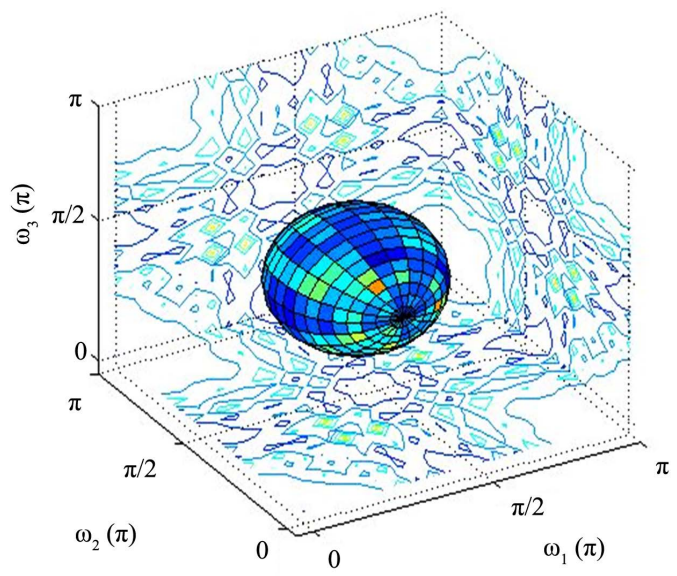

Figure 3. Trispectrum spherical representation. 


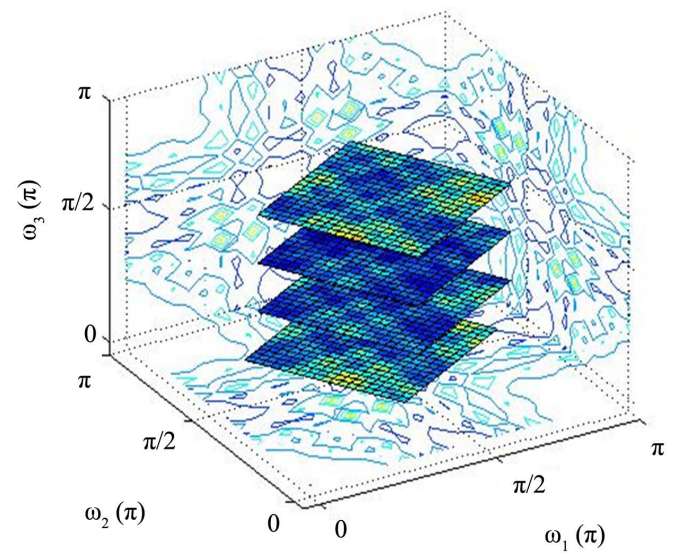

Figure 4. Trispectrum plane representation.

Fix a certain frequency coordinate of the three-frequency $\left(\omega_{1}, \omega_{2}, \omega_{3}\right)$ in the trispectrum, and then obtain a 2-D slice of the trispectrum. Let $\left(\omega_{1}, \omega_{2}\right)$ be variables and $\omega_{3}=\omega_{0}$ be a constant to get a 2-D slice of the trispectrum

$$
T S_{2 D}\left(\omega_{1}, \omega_{2}, \omega_{0}\right)=\gamma_{4 a} H\left(\omega_{1}\right) H\left(\omega_{2}\right) H\left(\omega_{0}\right) H^{*}\left(\omega_{1}+\omega_{2}+\omega_{0}\right)
$$

The 2-D slice of the normalized trispectrum is

$$
T_{c} S_{2 D}\left(\omega_{1}, \omega_{2}, \omega_{0}\right)=\frac{T S_{2 D}\left(\omega_{1}, \omega_{2}, \omega_{0}\right)}{\sqrt{\left|P\left(\omega_{1}\right) P\left(\omega_{2}\right) P\left(\omega_{0}\right) P\left(\omega_{1}+\omega_{2}+\omega_{0}\right)\right|}}
$$

The normalized amplitude expression is

$$
\begin{aligned}
& \left|T^{A R}\left(\omega_{1}, \omega_{2}, \omega_{0}\right)\right| \\
& =\frac{|K|}{\left|1+\sum_{i=1}^{p} \gamma_{i} \mathrm{e}^{-j i\left(\omega_{1}+\omega_{2}+\omega_{0}\right)}\right|\left|1+\sum_{i=1}^{p} \gamma_{4 a} \mathrm{e}^{-j i \omega_{1}}\right|\left|1+\sum_{i=1}^{p} \gamma_{4 a} \mathrm{e}^{-j i \omega_{2}}\right|\left|1+\sum_{i=1}^{p} \gamma_{4 a} \mathrm{e}^{-j i \omega_{0}}\right|}
\end{aligned}
$$

\section{Multi-Scale Decomposition of Two-Dimensional Wavelet}

Wavelet transform is a signal time-scale analysis method. It has the characteristics of multi-resolution analysis, and it has the ability to characterize the local characteristics of the signal in both the time and frequency domains. It is a kind of window whose size is fixed but its shape can be changed. Time-frequency localization analysis method with variable time window and frequency window.

The two-dimensional wavelet function is obtained through the tensor product transformation of the 21D wavelet function. The 2-D wavelet function decomposition is to decompose the low-frequency part of scale $j$ into four parts: the low-frequency part $a_{j}$ of scale $j+1$ and the high frequency part in three directions (horizontal, horizontal, Vertical, diagonal) $d_{j}^{1}, d_{j}^{2}, d_{j}^{3}$.

Suppose the original image has a total of $N \times N$ pixels, the scale parameter $j=$ 0 , the recursive decomposition is started from the original image, and the wavelet coefficient matrix $a_{j} d_{j}^{1}, d_{j}^{2}, d_{j}^{3}$ is decomposed at the $j$-th level, with $\left(2^{-j} N\right)^{2}$ pixels respectively. (The resolution is reduced $2^{-j}$ ). Since the factor $j$ 
represents the decomposition level and is related to the resolution of the image, $j$ is often called the resolution or decomposition scale. Taking the three-level wavelet decomposition of a 2 -d digital image as an example, the wavelet coefficient distribution is shown in Figure 5 [7].

\section{Analysis of Results}

First, the normalized amplitude-frequency characteristic diagram of the three-spectrum 2-d slice of the normal signal and the fault signal is obtained from the experimental data. The slice diagram is actually a 3-d figure of a 2-d array. In this paper, 1024 data are used to analyze each set of data. The 2-d slice is a 2-d array of $255 * 255$. Now three vibration states measured when the oil pressure is $1 \mathrm{MPa}, 3 \mathrm{MPa}$, and $5 \mathrm{MPa}$ are selected. For each of the 3 sets of data below, the 2-d slices and their corresponding wavelet packet coefficients are shown in Figures 6-9. The horizontal axis in the figure represents the frequency in $\mathrm{HZ}$, and the vertical axis represents the normalized amplitude, which is dimensionless. The 3-d graphics are shown in Figure 6 and Figure 8.

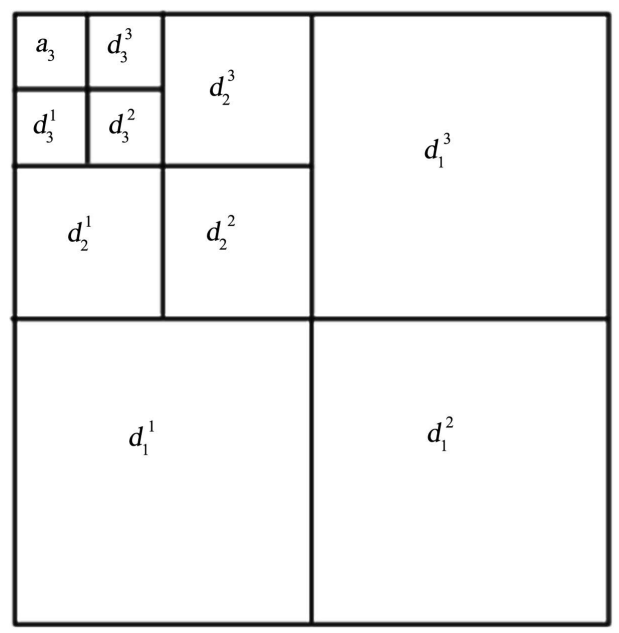

Figure 5. 2-d wavelet Multi-scale decomposition.
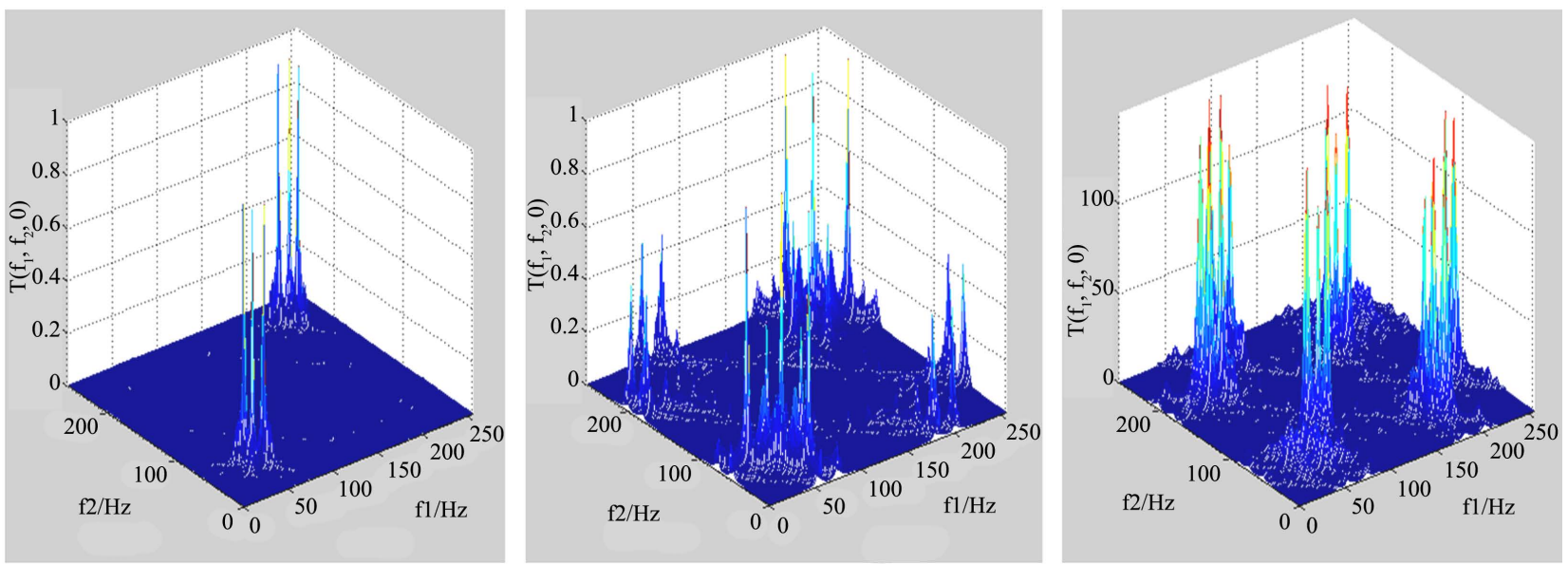

Figure 6. Two-dimensional slices of trispectrum in normal state. 

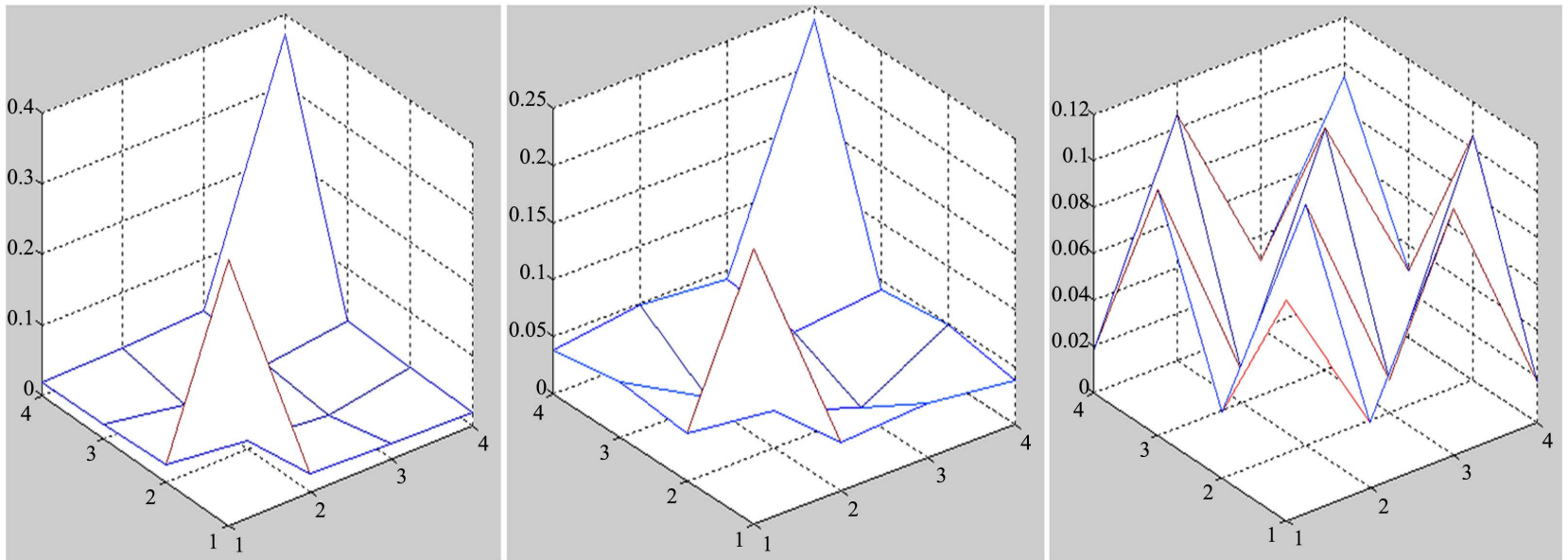

Figure 7. Wavelet decomposition low-frequency compression diagram in normal state.
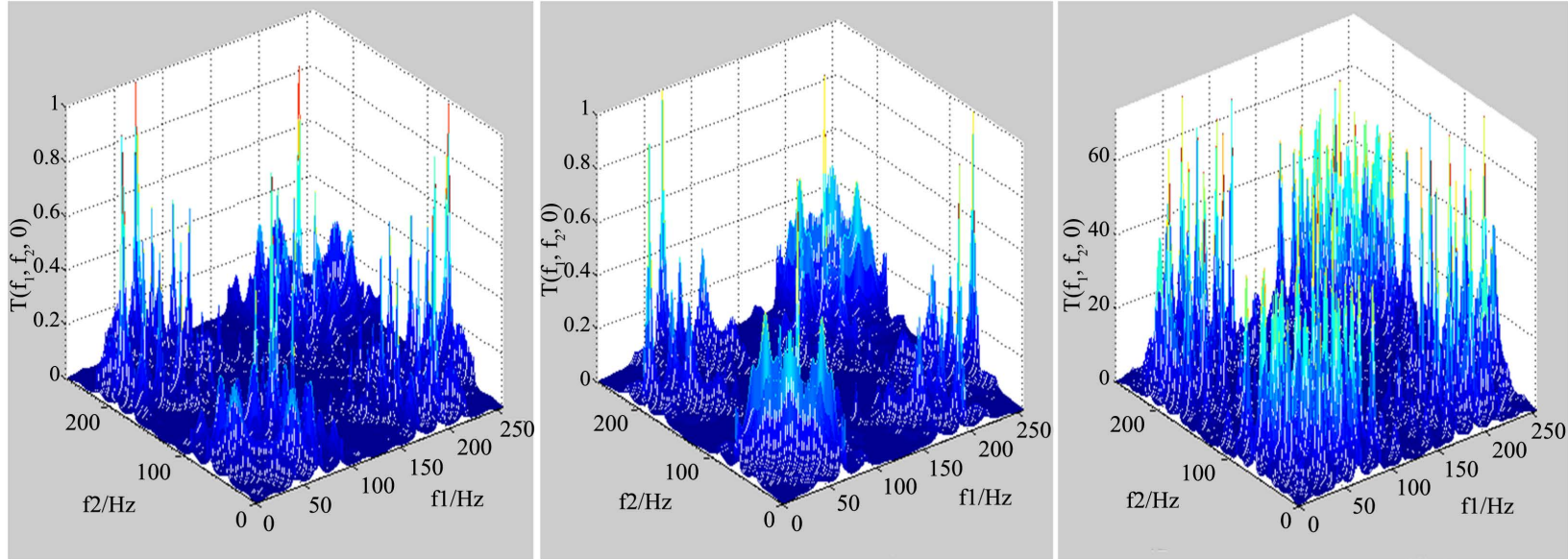

Figure 8. Two-dimensional slices of trispectrum in fault state.
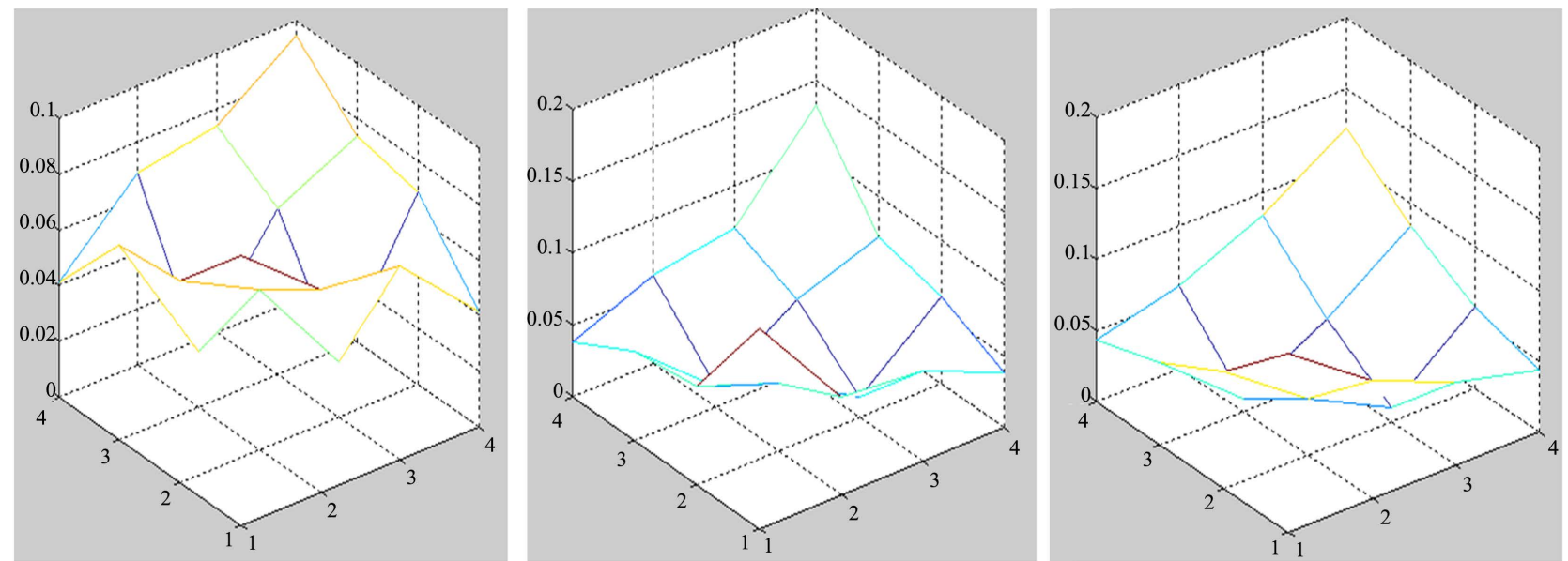

Figure 9. Wavelet decomposition low-frequency compression diagram in fault state.

It can be seen from Figure 6 and Figure 8 that no matter whether the hydraulic valve is in a normal state or a fault state, there are spectral peaks on the 2-d slice graph, and the amplitude is present and not zero, which clearly shows that 
the vibration signal of the hydraulic valve is nonlinear and non-Gaussian. In the normal state, the diagonal spectral peaks of the slice in the dual-frequency coordinate are flatter than in the fault state, and there are fewer peaks. In the case of a fault, there are more spectral peaks on the slice graph, and the spectral peaks are mixed and disordered situation. According to the intuitive difference between the 2-d slice diagram of the normal signal and the fault signal, the fault diagnosis can be performed based on the 2-d slice.

It can be seen from Figure 6 and Figure 8 that whether the hydraulic valve is in a normal state or a fault state, if the two-dimensional slice spectrum data is directly used to input the support vector machine for fault identification, one reason is that the amount of calculation is too large, the another is that the diagnostic results obtained through experiments are not ideal. Since the various frequency information contained in the original signal must be reflected in its high-order spectrum, and the wavelet packet can effectively extract these frequency information, this paper uses 2-d wavelet to perform feature extraction on each set of measured data. For comparative analysis, 6-level compression was performed on the original data, and the best diagnosis result was achieved.

Based on the symmetry of the 2-d slice spectrum, this article uses the db1 wavelet, and uses the compressed low-frequency coefficients as the input of LSSVM for fault identification. The compressed low-frequency coefficients are a $4 * 4$ two-dimensional array, and the graph is shown below the two-dimensional spectrum of the data, as shown in Figure 7 and Figure 9. In this experiment, 18 sets of normal data and 18 sets of fault data were measured under 5 different pressures and motor speeds. When training LSSVM, the compressed low-frequency coefficients are normalized. The method is to divide each data in a $4^{\star} 4$ two-dimensional array by the sum of all the data of the two-dimensional array. After normalization the data is shown in Table 1. Afterwards, the normal state and the fault state are coded as 1 and -1 respectively, and the corresponding 5 sets of data are taken out of normal data and fault data in Table 1 during training, and a total of 10 sets are used to train the established least squares support vector machine. After the training is completed, the LSSVM is used for fault identification. After parameter optimization and selection $\gamma=79, \sigma^{2}=1.88$, the trained network is used to simulate and identify the remaining 26 sets of data. The identification results are shown in Figure 10 [8].

Table 1. Features after normalization.

\begin{tabular}{|c|c|c|c|c|c|c|c|c|}
\hline \multirow{3}{*}{$\begin{array}{l}\text { Normal } \\
\text { state }\end{array}$} & 0.3751 & 0.0255 & 0.0207 & 0.0196 & 0.0255 & 0.0113 & 0.0012 & \\
\hline & 0.1459 & 0.0646 & 0.0665 & 0.0209 & 0.0646 & 0.0603 & 0.0111 & $\ldots \ldots$ \\
\hline & 0.2420 & 0.0424 & 0.0483 & 0.0380 & 0.0424 & 0.0324 & 0.0061 & ...... \\
\hline & & & & & & & & \\
\hline \multirow{3}{*}{$\begin{array}{l}\text { Fault } \\
\text { state }\end{array}$} & 0.1394 & 0.0683 & 0.0622 & 0.0383 & 0.0683 & 0.0476 & 0.0139 & $\ldots \ldots$ \\
\hline & 0.1477 & 0.0751 & 0.0577 & 0.0336 & 0.0751 & 0.0436 & 0.0104 & \\
\hline & 0.1066 & 0.0604 & 0.0775 & 0.0424 & 0.0604 & 0.0616 & 0.0127 & $\ldots \ldots$ \\
\hline
\end{tabular}




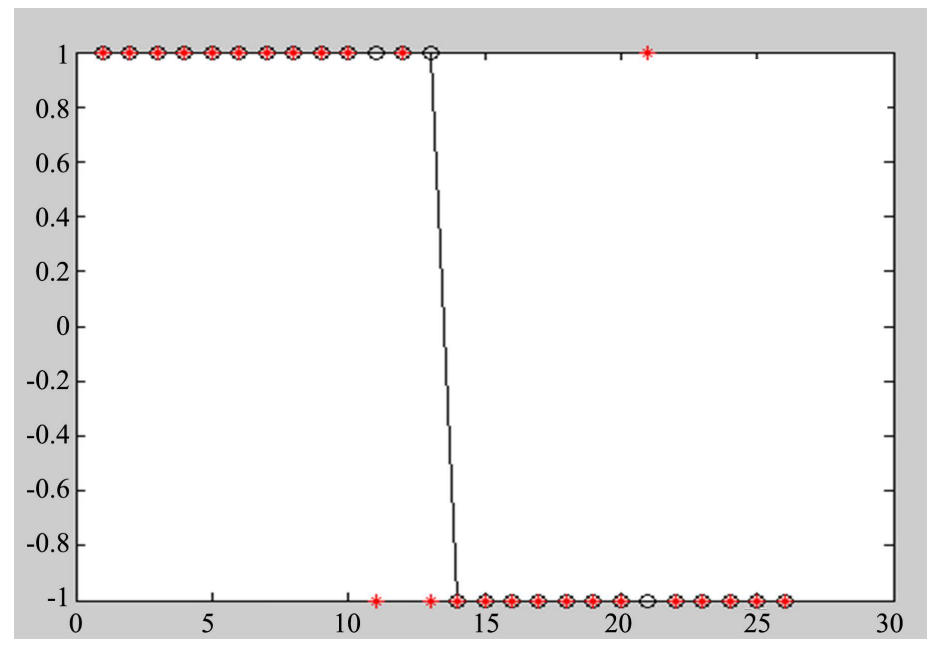

Figure 10. Simulation result.

The results show that there are only 2 sets in the normal data, and only 1 set in the fault data, a total of 3 sets of data which recognition errors, and the total recognition accuracy rate is close to $90 \%$ (excluding 10 groups of training data), indicating that the fault diagnosis method proposed in this paper is effective. Compared with bispectrum, trispectrum and its slices characterize random signals from a higher probability structure and provide an effective basis for analyzing non-Gaussian and nonlinear signals. It can not only qualitatively describe the linearity and nonlinearity of vibration signals, Gaussian and non-Gaussian performance, and can greatly improve the accuracy of mechanical fault diagnosis.

\section{Conclusion}

The high-order spectrum can effectively remove Gaussian noise, and the tri-spectrum and its slices represent random signals from a higher probability structure, providing an effective basis for analyzing non-Gaussian and nonlinear signals, and can greatly improve the accuracy of mechanical fault diagnosis. This paper uses two-dimensional wavelet multilevel decomposition to compress the two-dimensional array information of the three-spectrum two-dimensional slice, and then uses the least squares support vector machine to obtain the advantages of the global optimum under limited samples, and uses the compressed low-frequency coefficients as the input of LSSVM is used to diagnose the fault of the speed control valve, which has achieved good results. The author of this article will compare this fault diagnosis method with other methods in the follow-up work to prove the advantages of this fault diagnosis method and conduct theoretical discussions.

\section{Acknowledgements}

This paper is supported by Research Foundation of the Nanchang Normal University for Doctors (NSBSJJ2018014). Key R \& D Projects of Jiangxi Provincial 
Department of Science and Technology: 20192BBHL80002, 20192BBEL50040.

\section{Conflicts of Interest}

The author declares no conflicts of interest regarding the publication of this paper.

\section{References}

[1] Zhang, L.P. (2005) Principle, Use and Maintenance of Hydraulic Valve. Chemical Industry Press, Beijing, 146, 155, 204, 147-148.

[2] Liu, J.H., Jia, H.Q. and Ding, H. (2001) LabVIEW Tutorial of Graphical Programming Language for Virtual Instruments. Xidian University Press, Xi’an, 3-4.

[3] Cai, Q.Z. and Huang, Y.J. (2009) Study on Autoregressive Trispectrum Slices. Chinese Journal of Scientific Instrument, 30, 1-6.

[4] Chen, B.S. and Huang, Y.J. (2008) Study on Autoregressive Trispectrum and Its Slices. Chinese Control and Decision Conference, Yantai, 2-4 July 2008, 5124-5129. https://doi.org/10.1109/CCDC.2008.4598306

[5] Huang, Y.J., Liu, X.M. and Tian, T.C. (2006) AR Trispectral Characteristics of Electrological Actuator. Proceedings of the World Congress on Intelligent Control and Automation, 5782-5786.

[6] Raghuveer, M.R. (1990) Time-Domain Approaches to Quadratic Phase Coupling Estimation. IEEE Transaction on Automatic Control, 35, 48-56.

https://doi.org/10.1109/9.45142

[7] Nikravesh, Y., Rezaie, M.S., et al. (2019) Intelligent Fault Diagnosis of Bearings Based on Energy Levels in Frequency Bands Using Wavelet and Support Vector Machines (SVM). Journal of Manufacturing and Materials Processing, 3, 11.

[8] Sui, X., Wan, K. and Zhang, Y. (2019) Pattern Recognition of SEMG Based on Wavelet Packet Transform and Improved SVM. Optik, 176, 228-235.

https://doi.org/10.1016/j.ijleo.2018.09.040 